\title{
Orale HPV-Papillome unter hochaktiver antiretroviraler Therapie (HAART)
}

\author{
Oral HPV-Papillomas Under HAART
}

\section{Zusammenfassung}

Die Einführung der hochaktiven antiretroviralen Therapie (HAART) 1995 hat bisher bei HIV-infizierten Individuen zu einem deutlichen Rückgang der Morbidität wie auch Mortalität geführt. Insbesondere in den letzten Jahren hat sich die Lebensqualität vieler HIV-infizierter Patienten gebessert, durch Anstrengungen der Pharmaindustrie eine hocheffektive, antiretrovirale Therapie auch mit immer weniger Tabletten pro Tag möglich zu machen. Dennoch sind die unerwünschten Wirkungen dieser Therapie nicht unerheblich. Neben der z.T. deutlich stigmatisierenden Lipodystrophie, vor allem im Gesicht, ist das zunehmende Auftreten von oralen Viruspapillomen für die Patienten belastend. Darüber hinaus besteht die Gefahr, dass diese HPV-induzierten Papillome bei längerer Bestandsdauer maligne entarten können und orale Plattenepithelkarzinome möglicherweise die neuen HIV-assoziierten Tumore der „HAART-Ära“ werden. Die Therapie dieser Viruspapillome, deren deutliche Zunahme bisher in ihren Gründen völlig ungeklärt ist, ist ausgespochen hartnäckig und schwierig. Cidofovir, als bisher einziges HPVwirksames Virustatikum, stellt diesbezüglich eine gute therapeutische Option dar.

\section{Abstract}

The introduction of the highly active antiretroviral therapy (HAART) in 1995 has dramatically reduced the HIV-associated morbidity and mortality. Especially in the last few years, the quality of life for HIV-infected individuals has been improved by effective HAART with one or two tablets once or twice daily. However, the side effects of HAART are by no means negligible. Beside the stigmatising lipodystrophy, especially of the face, the increasing frequency of oral warts causes the patients additional stress. Moreover, these oral HPV-induced papillomas have the potential for malignant transformation. Squamous cell carcinoma of the oral mucosa may become the "tumours of the HAARTera". It is totally unclear why oral warts increase drastically in HIV-infected patients treated with HAART. However, clinicans are confronted with major therapeutic problems as a result. $\mathrm{Ci}$ dofovir, the only virostatic with proved action against HPV, may be a good therapeutical option.

\section{Einleitung}

Die breite Anwendung von HAART in den Industrieländern hat den zunächst aussichtslos wirkenden Kampf gegen die HIV-Infektion zu einer der größten Erfolgsgeschichten der wissenschaftlichen Medizin umgeschrieben. Mit der Einführung der Proteinasen-Inhibitoren (PI) 1995 wurde ein großer Durchbruch in der Therapie von HIV-infizierten Patienten erzielt, die bislang ausschließlich mit Medikamenten aus der Gruppe der Nukleosidischen Reverse-Transkriptase-Inhibitoren (NRTI) behandelt werden konnten. 1997 kam mit den Nicht-nukleosidischen Reverse-Transkriptase-Inhibitoren (NNRTI) eine bedeutende Alternative zu den PIs in der Kombinationstherapie hinzu. Die Kombination von NRTIs mit PIs oder NNRTIs sind bis heute die weitaus 


\section{H A A R T 2003, Entry-Inhibitoren \\ 1997, NNRTI, z.B. Efavirenz \\ 1995, PI, z.B. Saquinavir \\ 1987, NRTI, z.B. Zidovudin}

Abb. 1 Historische Entwicklung der antiretroviralen Therapie.

häufigsten und mit Studien in ihrer Wirksamkeit am besten abgesicherten HAART-Therapien. Im Jahr 2002 wurde mit Tenofovir das erste Medikament aus der Gruppe der Nukleotidischen Reverse-Transkriptase-Inhibitoren (NtRTI) eingeführt. Nur ein Jahr später konnte mit dem Medikament Enfuvirtide (T20) die Einführung des ersten Fusionsinhibitors aus der Klasse der so genannten Entry-Inhibitoren begrüßt werden (Abb.1). Somit stehen z.Zt. Medikamente aus drei verschiedenen Klassen, den Reverse-Transkriptase-Inhibitoren, den Proteinase-Inhibitoren sowie den Entry-Inhibitoren, zur Verfügung, die an jeweils verschiedenen Stellen die HIV-Replikation unterbinden können. Allein die Gruppe der Reverse-Transkriptase-Inhibitoren teilt sich in drei verschiedene Subgruppen mit z.Zt. 11 verschiedenen Medikamenten. Insgesamt stehen Mitte 2004 mindestestens 20 verschiedene Substanzen zur Behandlung der HIV-Infektion zur Verfügung (Tab.1).

Tab. 1 Anitretrovirale Medikamente

\begin{tabular}{|c|c|c|}
\hline $\begin{array}{l}\text { Reverse-Transkriptase- } \\
\text { Inhibitoren }\end{array}$ & Protease-Inhibitoren & Entry-Inhibitoren \\
\hline $\begin{array}{l}\text { NRTIs } \\
\text { Zidovudin (AZT) } \\
\text { Stavudin (d4T) } \\
\text { Didanosin (ddl) } \\
\text { Zalcitabin (ddC) } \\
\text { Lamivudin (3TC) } \\
\text { Abacavir (ABC) } \\
\text { Emtricitabin }\end{array}$ & $\begin{array}{l}\text { Saquinavir (SQVhg) } \\
\text { Saquinavir (SQVsg) } \\
\text { Ritonavir (RTV) } \\
\text { Indinavir (IDV) } \\
\text { Nelfinavir (NLV) } \\
\text { Amprenavir (APV) } \\
\text { Lopinavir/RTV (LPV) } \\
\text { Atazanavir (ATZ) }\end{array}$ & $\begin{array}{l}\text { Attachment-Inhibitoren. } \\
\text { Korezeptoren- } \\
\text { Antagonisten } \\
\text { Fusions-Inhibitoren } \\
\text { Enfurvitid (T20) }\end{array}$ \\
\hline $\begin{array}{l}\text { NNRTIs } \\
\text { Nevirapin (NVP) } \\
\text { Efavirenz (EFV) } \\
\text { Delavirdin (DLV) }\end{array}$ & & \\
\hline $\begin{array}{l}\text { NtRTI } \\
\text { Tenofovir (TFV) }\end{array}$ & & \\
\hline
\end{tabular}

In Folge von HAART kam es zu einem deutlichen Rückgang der HIV-bedingten Morbidität und Mortalität, auch in fortgeschrittenen Stadien der Infektion [1 - 3]. Verschiedene schwere opportunistische Infektionen, wie die Toxoplasmose, die CMV-Retinitis oder die Pneumozystis carinii-Pneumonie (PCP), haben heute selbst in HIV-spezialisierten Abteilungen Seltenheitswert. Das Gleiche gilt für das HIV-assoziierte Kaposi-Sarkom (KS), das unter HAART viel rarer geworden ist. In der Regel wird ein KS nur noch diagnostiziert, wenn gleichzeitig die Erstdiagnose einer
HIV-Infektion festgestellt wird und die Patienten noch nicht unter HAART stehen. Ferner hat die Prävalenz von anderen HIV-assoziierten Dermatosen, wie z.B. der oralen Haarleukoplakie, der schweren exsudativen Psoriasis vulgaris oder dem ausgedehnten seborrhoischen Ekzem, deutlich abgenommen [4,5].

Neben diesen großen Erfolgen von HAART scheinen die unerwünschten Nebenwirkungen der antiretroviralen Kombinationstherapie zu verblassen. Unter der dermatologischen Perspektive haben insbesondere allergische Arzneimittelexantheme zugenommen und zeigen sich eine Reihe weiterer kutaner Nebenwirkungen (Retinoidartige Dermatitis, Pruritus, Ungius incanatus, Paronychien etc.) der antiretroviralen Therapie [6,7]. Daneben kommt es bei mindestens $60 \%$ der Patienten unter HAART zu dem so genannten Lipodystrophiesyndrom (LDS). Neben verschiedenen metabolischen Veränderungen (Hyperlipidämie, Diabetes mellitus, etc.) können z.B. eine massive Abnahme des subkutanen Fettgewebes, insbesondere im Gesicht und an den Extremitäten (Abb. 2), sowie die Entwicklung von lokalisierten Fettpolstern im Bereich des Nackens („,buffalo hump“) und abdominell („crixi-belly“) beobachtet werden. Diese deutlich sichtbaren Veränderungen, speziell das Verschwinden des subkutanen Fettgewebes im Gesichtsbereich, werden von den Patienten als stigmatisierend und ihre Lebensqualität erheblich beeinträchtigend empfunden.
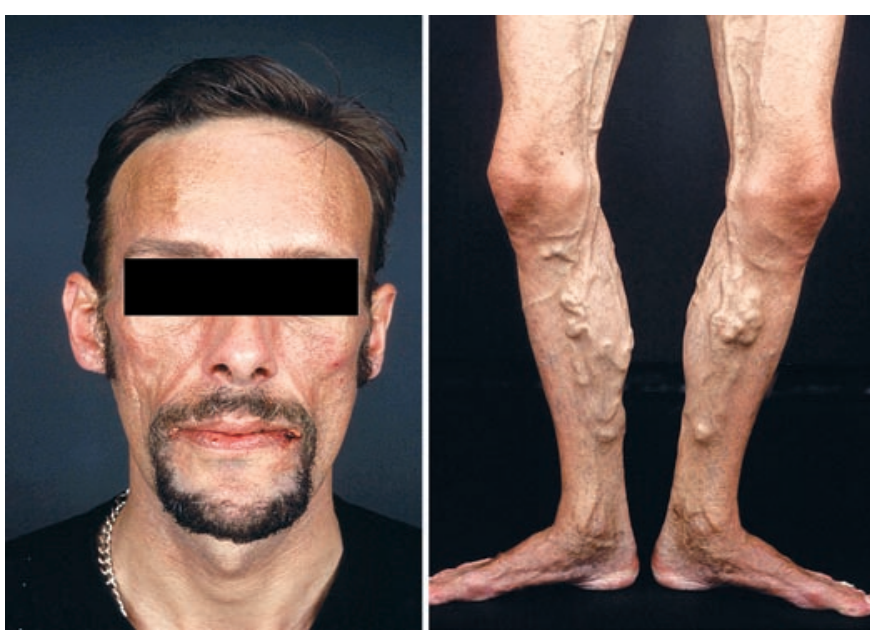

Abb. 2 Lipodystrophie unter HAART mit deutlicher Abnahme des subkutanen Fettgewebes.

Ein weiteres Phänomen, welches in diesem Ausmaß erst unter HAART beobachtet wurde, ist die auffällige Zunahme von humanen Papillomvirus (HPV)-induzierten Haut- und Schleimhautveränderungen, insbesondere im Mundschleimhautbereich.

\section{Prävalenz von oralen HPV-Papillomen bei HIV-infizierten Patienten}

Die Prävalenz oraler Viruspapillome ist in der immunkompetenten Allgemeinbevölkerung ausgesprochen niedrig und wird mit weniger als 0,5\% angegeben [8]. Bei iatrogen Immunsupprimierten liegt die Prävalenz bei ca. 0,5-1\%. Bei HIV-infizierten Erwachsenen liegt die Prävalenz oraler HPV-Papillome deutlich 
höher mit mehr als 2,5\% der Betroffenen [9-11]. Greenspan und Mitarbeiter [11] untersuchten retrospektiv an 1300 HIV-Patienten eines spezialisierten Zentrums für Mundschleimhauterkrankungen in San Francisco die Prävalenz opportunistischer Infektionen im Mundraum. Während einer Reihe von Erkrankungen, wie z.B. die orale Kandidose, das orale KS oder die orale Haarleukoplakie, stark abnahmen, zeigte sich ein deutlicher Anstieg von oralen HPV-Papillomen im Untersuchungszeitraum zwischen 1990-1999. Entscheidend war, dass die Prävalenz für orale Viruspapillome bei 5\% für Patienten ohne antiretrovirale Therapie lag, während die Prävalenz für Patienten mit HAART inklusive PIs $23 \%$ betrug. Auch in dem eigenen Patientenkollektiv der Klinik und Hochschulambulanz für Dermatologie am Campus Benjamin Franklin, Charité, zeigt sich eine stetige Zunahme von Patienten mit oralen Viruspapillomen, während z.B. die KS-Fälle deutlich abgenommen haben.

Die Ursachen für dieses Phänomen sind bisher völlig ungeklärt. Als Ausdruck eines Immunrekonstitutionsphänomens, wie es bei der Tuberkulose, der CMV-Retinitis oder Zoster gelegentlich beobachtet wurde, werteten einige Autoren den Anstieg von oralen HPV-Papillomen [11,12]. Inwieweit die Zunahme von ungeschütztem Geschlechtsverkehr unter HAART, der sich insbesondere durch den deutlichen Anstieg von Lues- und Gonorrhoefällen unter dem homosexuellen Patientenkollektiv manifestiert, auch eine Erklärung für die Verbreitung oraler HPV-Papillome ist, kann nur spekuliert werden.

\section{Klinik und HPV-Subtypen oraler HPV-Papillome}

Klinisch imponieren diese HPV-Papillome als zumeist erythematös-weißliche, papulöse Hautveränderungen mit papillomatöser, z.T. auch verruciformer Oberfläche, die einzelstehend (Abb.3a) oder beetartig (Abb. 3b) im Bereich der gesamten Mundschleimhaut zu finden sind. Differenzialdiagnostisch müssen die fokale epitheliale Hyperplasie (Morbus Heck), die floride orale Papillomatose, die orale Haarleukoplakie, eine atypische Kandida- oder HSV-Infektion, eine Tabak-induzierte Leukoplakie oder ein Plattenepithelkarzinom abgegrenzt werden [13]. Zur diagnostischen Sicherung oraler HPV-Papillome sollte trotz der meist charakteristischen Klinik eine Biopsie erfolgen. Dabei zeigen sich in der Histologie typischerweise Koilozyten sowie verstärkt Keratohyalingranula in den oberen Schichten der Mukosa und eine Hyperkeratose mit Parakeratosekegeln. Ferner gehört der Nachweis von HPV, heute zumeist mit immunhistologischen oder molekularbiologischen Methoden, wie z.B. der Polymerase-Kettenreaktion (PCR), zur differenzialdiagnostischen Abklärung dazu.

Interessanterweise scheint die Prävalenz von HPV auch in klinisch unauffälliger Mundschleimhaut ausgesprochen hoch zu sein. Terai und Mitarbeiter [14] konnten bei ca. $80 \%$ von immunkompetenten Individuen mit klinisch unauffälliger Mundschleimhaut HPV nachweisen Dabei zeigten sich am häufigsten die Subtypen HPV-16 und -18, die im Genitoanalbereich als Typen mit höherer maligner Potenz gelten. Bei HIV-infizierten Patienten wurden verschiedenste Typen von HPV in oralen Viruspapillomen gefunden [15]. In der Untersuchung von Völter und Mitarbeitern [15] an 67 HIV-infizierten Patienten konnten insbesondere die HPV-Typen 7 mit 19\% und 32 mit 28\% häufig nach-
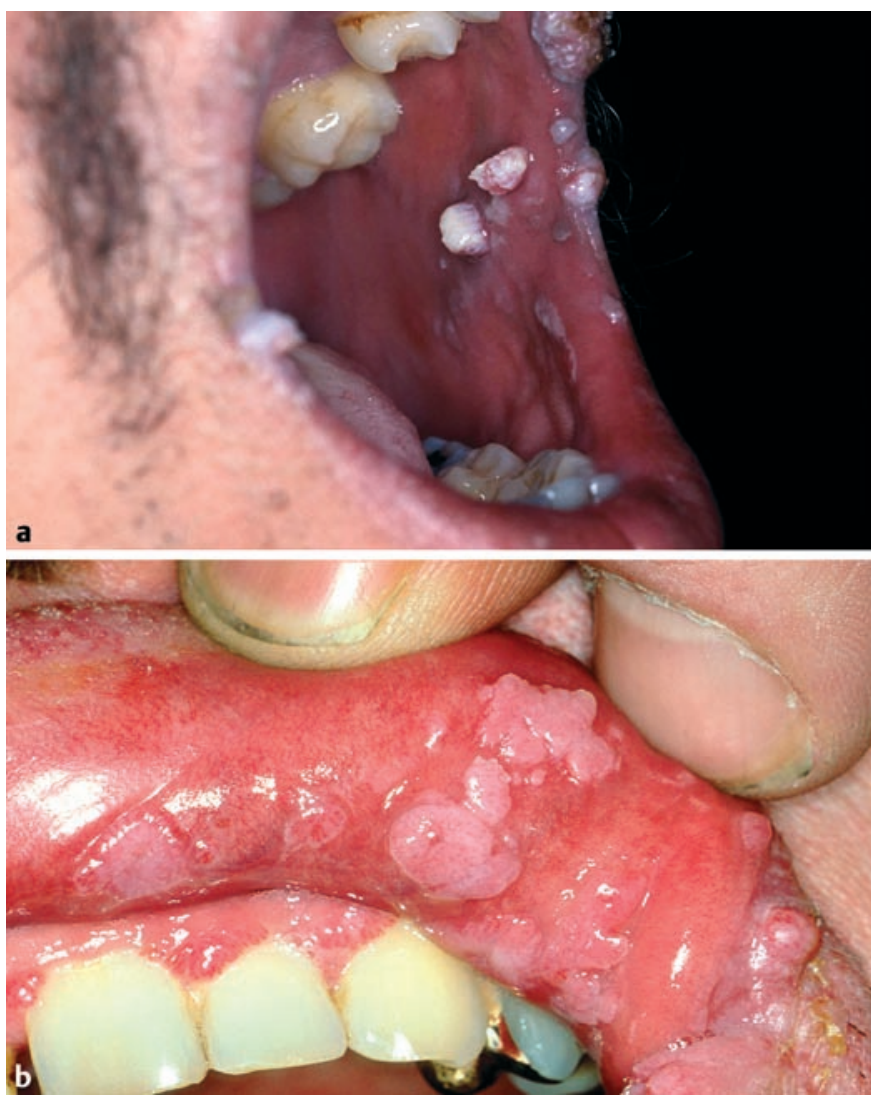

Abb. 3 a,b Klinische Manifestation von oralen Viruspapillomen.

gewiesen werden. Der HPV-Typ 7 wird normalerweise bei den so genannten „Schlachterwarzen“ gefunden, d.h. vor allem bei Menschen, die in der Fleischverarbeitung tätig sind. Diese „Schlachterwarzen“ zeigen sich als verrucöse Hautveränderungen insbesondere im Bereich der Finger. Die häufige Beobachtung dieses Typs unter HIV-Patienten ist bemerkenswert. Die Assoziation zwischen HPV-Typen und malignen oder prämalignen Läsionen im Bereich der Mundschleimhaut ist bisher noch unklar. Ob die mit erhöhtem onkogenem Risiko im Genitoanalschleimhautbereich assoziierten HPV-Subtypen, z.B. 16, 18, 30, 31, 33, auch mit vermehrter Malignombildung im Bereich der Mundschleimhaut assoziiert sind, bleibt z.Zt. noch ungeklärt. In jedem Falle können die Viruspapillome auch im Bereich der Mundschleimhaut bei HIV-Patienten entarten. Die Entwicklung von Plattenepithelkarzinomen der oralen Mukosa in dieser Patientengruppe ist bereits beschrieben worden [16]. Auch ein von uns betreuter, unter langjähriger HAART-Therapie stehender Patient hat im Bereich der Mundschleimhaut Plattenepithelkarzinome entwickelt. Diese entstanden auf dem Boden von HPV-33und HPV-72-positiven enoralen Viruspapillomen. Bemerkenswert ist, dass dieser Patienten zuvor bei rezidivierenden genitoanalen Condylomata acuminata ein Analkarzinom entwickelt hatte. Eine Zunnahme von Malignomen im Bereich der Mundschleimhaut, ausgehend von langjährig bestehenden HPV-Papillomen, muss bei HIV-infizierten Patienten befürchtet werden. 

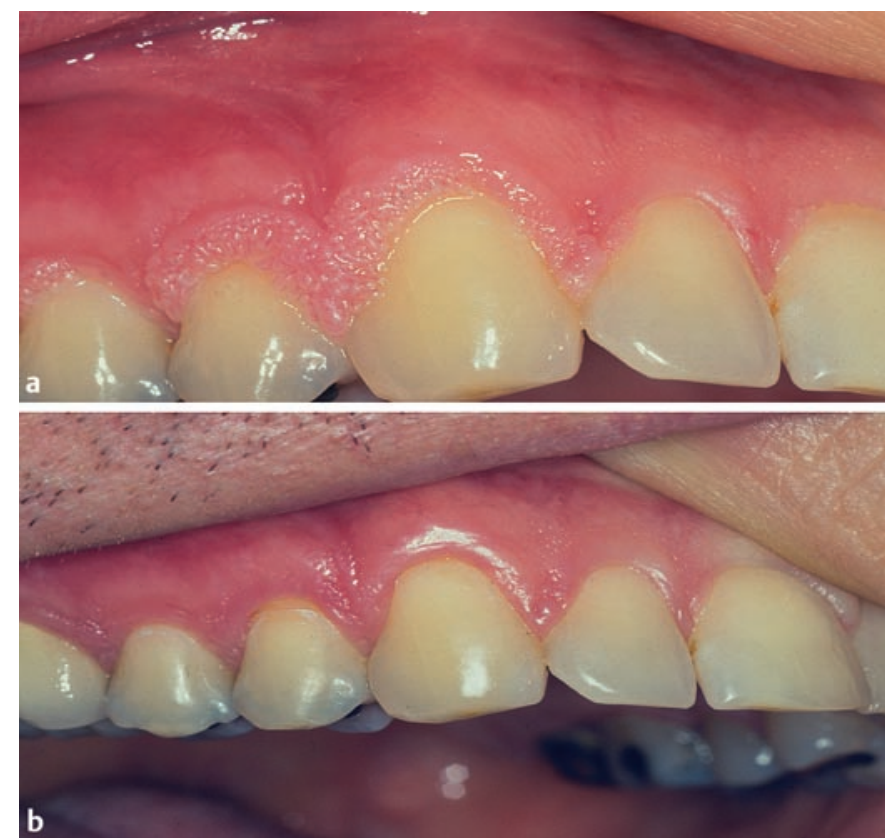

Abb. 4 Orale Viruspapillome vor (a) und 11 Wochen nach (b) Therapie mit Cidofovir 1\%-Lösung.

Therapie von oralen HPV-Papillomen bei HIV-infizierten Patienten

Klassischerweise werden orale HPV-Papillome vor allem mit operativen Methoden wie z.B. der $\mathrm{CO}_{2}$-Laserablation, der chirurgischen Exzision oder der Elektrokauterisation behandelt. Nachteilig an diesen verschiedenen operativen Therapieverfahren ist, dass sie häufig nur in Intubationsnarkose möglich und bei Viruspapillomen im Bereich der Interdentalregion nur schwierig durchzuführen sind. Bei der starken Vaskularisierung der Mundschleimhaut kommt es schnell zu Blutungen und damit zu der Gefahr der Verbreitung von HIV und HPV im Operationsgebiet. Insofern zeigt sich eine hohe Rezidivrate.

Neben dem operativen Verfahren wurde kasuistisch über verschiedene Therapiemethoden mit lokal oder intraläsional applizierten Medikamenten berichtet, wie z.B. Podophylotoxin, 5-Fluoruracil, Interferon-alpha/-beta, Bleomycin und Imiquimod $[17,18,26]$. Bei ausgeprägten Befunden ist diese intraläsionale oder lokale Behandlung häufig nur eingeschränkt möglich, und es wird z.T. über starke, lokale Reizungen berichtet. Insbesondere die Anwendung von intraläsional zugefügtem Bleomycin ist darüber hinaus äußerst schmerzhaft. Auch systemische Therapien zur Behandlung von oralen Warzen bei HIV-infizierten Patienten mit z.B. Interferon-alpha oder Cimetitdin sind kasuistisch als erfolgreich berichtet worden [19].

\section{Cidofovir in der Behandlung von oralen HPV-Papillomen}

Cidofovir ist ein azyklisches Nukleositphosphonatanalogon, das gegen ein breites Spektrum von DNA-Viren (z.B. Herpesviren, Adenoviren, Polyomaviren und Papillomaviren) als auch gegen Poxviren und Retroviren wirkt [20]. Cidofovir ist somit z.Zt. das einzige Virustatikum mit einer nachgewiesenen Wirksamkeit gegen HPV. Der genaue Mechanismus ist bisher noch unklar. Mit Sicherheit hat Cidofovir einen antiviralen und antiproliferativen Effekt auf die rasch teilenden HPV-haltigen Epithelzellen [21]. Cidofovir ist für die i.v. Therapie der CMV-Retinitis bei HIVinfizierten Patienten zugelassen. Bereits mehrfach ist über seinen erfolgreichen Einsatz in der Behandlung von rezidivierenden Genitoanalwarzen bei HIV-infizierten Patienten berichtet worden $[22,23]$. Neben der topischen Anwendung von Cidofovir kam auch die Kombination mit chirurgischen Maßnahmen zur Behandlung von HPV-Papillomen im Genitalbereich erfolgreich zur Anwendung [24]. In der Klinik und Hochschulambulanz für Dermatologie, Charité, Campus Benjamin Franklin, wurden bereits mehrere HIV-infizierte Patienten mit therapierefraktären, oralen Viruspapillomen mit 1\%- oder 3\%-iger Cidofovir-Lösung mit großem Erfolg therapiert [25]. Dabei erhielten die Patienten eine $1 \%$ - oder $3 \%$-ige Cidofovir-Lösung, die sie $1 \times$ täglich auf die betroffenen Areale auftupften für 3-12 Wochen Abb. 4. Neben milden bis mittelschweren Schleimhautreizungen bis hin zur Erosion, die gelegentlich zu einer Therapieunterbrechung zwangen, konnten keine wesentlichen Nebenwirkungen, insbesondere keine nephrotoxischen Nebenwirkungen festgestellt werden. Somit scheint die Behandlung von oralen Warzen mit lokal applizierter Cidofovir-Lösung eine gute therapeutische Option zu sein. Rezidive können auch unter dieser Therapie auftreten, sprechen jedoch prompt auf erneute Therapieeinleitung an.

\section{Literatur}

${ }^{1}$ Brodt HR, Kamps BS, Gute P et al. Changing incidence of AIDS-defining illnesses in the era of antiretroviral combination therapy. AIDS 1997; 11: $1731-1738$

2 Palella FJ, Jr., Delaney KM, Moorman AC et al. Declining morbidity and mortality among patients with advanced human immunodeficiency virus infection. HIV Outpatient Study Investigators. N Engl J Med 1998; 338: $853-860$

${ }^{3}$ Mocroft A, Katlama C, Johnson AM et al. AIDS across Europe, 1994-98: the EuroSIDA study. Lancet 2000; 356: 291 -296

${ }^{4}$ Costner M, Cockerell CJ. The changing spectrum of the cutaneous manifestations of HIV disease. Arch Dermatol 1998; 134: 1290 - 1292

${ }^{5}$ Husak R, Lüke C, Goerdt S, Orfanos CE. Veränderungen in der Prävalenz HIV-assoziierter Dermatosen. Hautarzt 1999; 50: 149

${ }^{6}$ Garcia-Silva J, Almagro M, Juega J et al. Protease inhibitor-related paronychia, ingrown toenails, desquamative cheilitis and cutaneous xerosis. AIDS 2000; 14: 1289 - 1291

${ }^{7}$ Ward HA, Russo GG, Shrum J. Cutaneous manifestations of antiretroviral therapy. J Am Acad Dermatol 2002; 46: 284-293

${ }^{8}$ Bouquot JE, Gundlach KK. Oral exophytic lesions in 23,616 white Americans over 35 years of age. Oral Surg Oral Med Oral Pathol 1986; 62: $284-291$

${ }^{9}$ King MD, Reznik DA, O’Daniels CM et al. Human papillomavirus-associated orals warts among human immunodeficiency virus-seropositive patients in the era of highly active antiretroviral therapy: an emerging infection. Clin Infect Dis 2002; 34: 641-648

${ }^{10}$ Patton LL, McKaig R, Strauss R et al. Changing prevalence of oral manifestations of human immuno-deficiency virus in the era of protease inhibitor therapy. Oral Surg Oral Med Oral Pathol Oral Radiol Endod 2000; 89: 299-304

${ }^{11}$ Greenspan D, Canchola AJ, MacPhail LA et al. Effect of highly active antiretroviral therapy on frequency of oral warts. Lancet 2001; 357: $1411-1412$

12 Birnbaum W, Hodgson TA, Reichart PA et al. Prognostic significance of HIV-associated oral lesions and their relation to therapy. Oral Diseases 2002; 8 (Suppl. 2): 110-114

${ }^{13}$ Husak R, Tebbe B, Goerdt S, Wölfer LU et al. Pseudotumour of the tongue caused by herpes simplex virus type 2 in an HIV- 1 infected immunosuppressed patient. Br J Dermatol 1998; 139: 118-121 
${ }^{14}$ Terai M, Hashimoto K, Yoda K, Sata T. High prevalence of human papillomaviruses in the normal oral cavity of adults. Oral Microbiol Immunol 1999; 14: $201-205$

${ }^{15}$ Völter C, He Y, Delius H et al. Novel HPV types present in oral papillomatous lesions from patients with HIV infection. Int J Cancer 1996; 66: $453-456$

${ }^{16}$ Piattelli A, Rubini C, Fioroni M, Iezzi T. Warty carcinoma of the oral mucosa in an HIV+ patient. Oral Oncology 2001; 37: 665 - 667

17 Beutner KR, Spruance SL, Hougham AJ et al. Treatment of genital warts with an immune response modifier (imiquimod). J Am Acad Dermatol 1998; 38: $230-239$

18 Girao L, Franca I, Macedo H et al. Treatment of oral condylomata acuminata in a HIV-1 patient with bleomycin. JEADV 2000; 14: 321 - 322

${ }^{19}$ Wargon O. Cimetidine for mucosal warts in an HIV positive adult. Australas J Dermatol 1996; 37: 149-150

${ }^{20}$ De Clerq E. Acrylic nucleotide phosphonates in the chemotherapy of DNA virus and retrovirus infections. Intervirology 1997; 40: 295 - 303
${ }^{21}$ Andrei G, Snoeck R, Piette J et al. Antiproliferative effects of acyclic nucleoside phosphonate on human (HPV)-harboring cell lines compared with HPV-negative cell lines. Oncol Res 1998; 10: 523-531

22 Hengge UR, Tietze G. Successful treatment of recalcitrant condyloma with topical cidofovir. Sex Transm Inf 2000; 76: 143

${ }^{23}$ Martinelli C, Farese A, Mistro AD et al. Resolution of recurrent perianal condylomata acuminata by topical cidofovir in patients with HIV infection. J Eur Acad Dermatol Venereol 2001; 15: 568 - 569

${ }^{24}$ Orlando G, Fasolo MM, Beretta R et al. Combined surgery and cidofovir is an effective treatment for genital warts in HIV-infected patients. AIDS 2002; 15: $447-450$

${ }^{25}$ Husak R, Zouboulis CC, Sander-Bähr C et al. Refractory oral HPV papillomas treatet topically with 1-3\% Cidofovir solutions in HIV-1-infected patients. $\mathrm{Br} \mathrm{J}$ Dermatol: in press

${ }^{26}$ Lozada-Nur F, Glick M, Schubert M, Silverberg I. Use of intralesional interferon-alpha for the treatment of recalcitrant oral warts in patients with AIDS: a report of 4 cases. Oral Surg Oral Med Oral Pathol Oral Radiol Endod 2001; 92: 617-622

\section{Buchbesprechung}

\section{Dermatokosmetik \\ Martina Kerscher}

Darmstadt: Steinkopff, 2004. 168 S., 51 Abb. Geb. 84,95€.

ISBN 3-7985-1455-0

Medizingeschichte ist Teil der Kulturgeschichte und die Dokumentationen und Zeugnisse stammen aus den gleichen frühen Zeiten schon. Krankheiten, Lebensbrüche und der Tod wurden göttlicher Ungnade oder Strafe zugeordnet, denen in sakralen Handlungen zu begegnen ist. Den „Bann zu lösen“ obliegt dem Priester. Selbstheilende Krankheiten und solche mit zeitlicher Begrenzung sind neben der Begleitung Sterbender von besonderer Bedeutung für eingeweihte Heiler, die sich zunehmend spezialisieren. Ist der Krankheitsablauf erkannt, sind die Heilungsriten so festzulegen, dass sie zur richtigen Zeit Wirkung versprechen, als Beweis für die Allmacht der Gottheit und auch für die spezielle Beziehung des Heilers zu dieser. Heilmittel aus der Natur, Pflanzen, Mineralien und tierische Produkte wurden erkannt und angewandt, mit Wirkung und mit Symboleffekt. Damals waren Medizin und Kosmetik noch zusammen, die dekorative, die verdeckende und auch die schmückende. Aber auch Insignien der Übergänge und der Zugehörigkeit; Rituale der Rangordnung sind oft dauerhaft, dekorativ und gültig. Erst viel später haben sich Heiler von Priestern geschieden und die Medizin von der
Kosmetik gesondert. In den letzten Jahrzehnten nun kommen die naturwissenschaftliche Dermatologie und die rationale, wirkungsorientierte Kosmetologie wieder zusammen, gut und fest, hilfreich und effektiv. Der Bemühungen sind viele. Die Menschen danken's beiden Bereichen.

Dies ist auch der Tenor des bemerkenswerten Buches aus dem Studiengang Kosmetik und Körperpflege der Universität Hamburg, vorgelegt von Frau Prof. Martina Kerscher, einer ausgewiesenen Dermatologin, unter Mitarbeit von Frau Dr. Stefanie Williams. Eine Brücke soll es sein, und eine Verklammerung im besten Sinn ist es geworden. Klug eingeteilt, präzise ausgesucht und lesefreundlich dargestellt, wird das Buch mit 162 Seiten und vielen Abbildungen und Tabellen dem Anliegen bestens gerecht. In 5 Kapiteln wird die Haut in ihrer Vielfalt und Eigenart vorgestellt, und in 4 Kapitel wird die kosmetische Seite eingebracht: Lokal wirkende Kosmetika, Botulinumtoxin, injizierbare Implantate und chemisches Peeling sind die Schwerpunkte. Klar werden die Möglichkeiten, die Grenzen, die Techniken und die rechtlichen Umstände vorgestellt, kritisch durchdacht, verständlich und ohne nebulöse Wirrversuche, dafür immer auf den Evidenzen basierend. Erfreulich und gut zu lesen, wird das Buch helfen, gut zu helfen.

E. G. Jung, Heidelberg 